
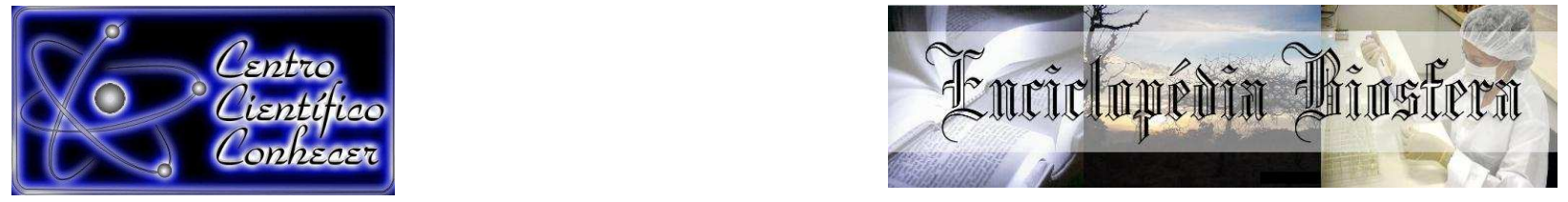

\title{
MEGAESÔFAGO ADQUIRIDO SECUNDÁRIO À PERSISTÊNCIA DO QUARTO ARCO AÓRTICO DIREITO EM CÃES DAS RAÇAS PASTOR ALEMÃO E PASTOR CANADENSE: RELATO DE CASOS
}

\author{
Rômulo Ferreira de Assumção ${ }^{1}$, Gabriele Barros Mothé $^{2}$, Natasha Nogueira \\ Ferreira $^{3}$, Paloma Helena Sanches da Silva ${ }^{4}$, Ana Maria Barros Soares ${ }^{5}$ \\ ${ }^{1}$ Mestrando da área de Fisiopatologia e Ciências Cirúrgicas da Universidade do Estado do Rio de \\ Janeiro - UERJ, Rio de Janeiro, RJ, Brasil. Autor para correspondência: \\ mvromuloferreira@gmail.com \\ ${ }^{2}$ Residente da área de Clínica Médica de Pequenos Animais do Programa de Residência em \\ Medicina Veterinária da Universidade Federal Fluminense - UFF, Niterói, RJ, Brasil. \\ ${ }^{3}$ Residente da área de Clínica Cirúrgica de Pequenos Animais do Programa de Residência em \\ Medicina Veterinária da Universidade Federal Fluminense - UFF, Niterói, RJ, Brasil. \\ ${ }^{4}$ Médica veterinária autônoma, Rio de Janeiro, RJ, Brasil. \\ ${ }^{5}$ Docente da Faculdade de Medicina Veterinária do Departamento de Patologia e Clínica Veterinária \\ da Universidade Federal Fluminense, Niterói, RJ, Brasil.
}

Recebido em: 03/10/2016 - Aprovado em: 21/11/2016 - Publicado em: 05/12/2016 DOI: 10.18677/EnciBio_2016B_065

\begin{abstract}
RESUMO
O objetivo do trabalho é relatar a ocorrência de megaesôfago secundário associado à persistência do quarto arco aórtico direito em dois cães das raças pastor alemão e pastor canadense, cujo manejo clínico tardio não apresentou resultados satisfatórios, mostrando a necessidade da correção cirúrgica precoce. As anomalias dos anéis vasculares são malformações congênitas dos principais vasos da base do coração que podem ocasionar o envolvimento e constrição parcial ou completa do esôfago ou da traqueia. Entre essas anomalias, a persistência do quarto arco aórtico direito, a mais frequente em cães, pode resultar em alterações digestivas, como o megaesôfago. Neste trabalho, são descritos dois casos de megaesôfago secundário à persistência de arco aórtico direito em cães com dois meses de idade, atendidos no Hospital Universitário de Medicina Veterinária da Universidade Federal Fluminense (HUVET-UFF). A sintomatologia clínica aliada ao esofagograma contrastado permitiu o diagnóstico de anomalia do anel vascular e, por conseguinte, do megaesôfago. Em ambos os casos foi identificada pneumonia por aspiração do conteúdo regurgitado. O procedimento cirúrgico é a única possibilidade de sobrevida para pacientes acometidos por essa enfermidade e deve ser instituído o mais precocemente possível, uma vez que o tratamento conservador não é suficiente para a recuperação do paciente e a garantia da qualidade de vida.
\end{abstract}

PALAVRAS-CHAVE: anomalia de anel vascular, canino, regurgitação.

\section{SECONDARY MEGAESOPHAGUS ASSOCIATED TO PERSISTENT FOURTH RIGHT AORTIC ARCH IN DOGS OF GERMAN SHEPHERD AND CANADIAN SHEPHERD BREEDS: CASE REPORTS}

\footnotetext{
ABSTRACT

The aim of this study is to report the occurrence of secondary megaesophagus associated to persistent fourth right aortic arch in two dogs of German shepherd and ENCICLOPÉDIA BIOSFERA, Centro Científico Conhecer - Goiânia, v.13 n.24; p. 693 2016
} 
Canadian shepherd breeds, whose late clinical treatment did not present satisfatory results, showing the need for early surgical correction. The anomalies of vascular rings are congenital malformations of the main vessels of the heart that may result in the envolvement and partial or complete constriction of the esophagus and/or trachea. Among these anomalies, the persistence fourth right aortic arch, the most common in dogs, may result in digestive disorders such as megaesophagus. In this report, we describe two cases of megaesophagus secondary to persistent fourth right aortic arch in two-month old dogs referred to University Hospital of Veterinary Medicine, Universidade Federal Fluminense (HUVET-UFF) for assistance. The clinical symptomatology combined to contrast esophagogram allowed the diagnosis of anomaly of vascular ring and the resulting megaesophagus. In both cases, aspiration pneumonia of regurgitated food was identified. Surgical procedure is the only possibility of survival for patients suffering from this disease and must be performed as soon as possible, since conservative treatment is not sufficient to guarantee the recovery of the patient and to ensure the quality of life of these animals.

KEYWORDS: Canine, Regurgitation, Vascular ring anomaly

\section{INTRODUÇÃO}

As desordens gastrointestinais apresentam grande prevalência e são reconhecidas como um dos motivos mais comuns de consulta na clínica veterinária, refletindo-se na casuística dos atendimentos de pequenos animais (DIAZ et al., 2012). Entre estas desordens, existem aquelas que têm relação com a fase de deglutição dos alimentos, envolvendo principalmente o esôfago, cuja função é o transporte de líquidos e sólidos ingeridos da cavidade oral até o estômago (ETTINGER \& FELDMAN, 2004).

Quando o esôfago apresenta alguma alteração que o impede de exercer sua função adequadamente, os sinais clínicos mais frequentes são odinofagia, disfagia, sialorréia e, principalmente, regurgitação (GELBERG, 2012; KYLES, 2012; WASHABAU, 2013). O megaesôfago é a principal causa de regurgitação em cães e consiste em dilatação do esôfago e peristalse esofágica ineficiente (GELBERG, 2012; KYLES, 2012). Pode ocorrer de forma secundária adquirida em diversas condições, entre elas a persistência do arco aórtico direito (PAAD) (KYLES, 2012; WASHABAU, 2013).

As anomalias de anel vascular, como a PAAD, são malformações congênitas dos principais vasos da base do coração que, por estarem em posições alteradas, comprimem o esôfago ou a traqueia, promovendo a obstrução física desses órgãos e provocando alterações digestivas ou respiratórias (MENZEL \& DISTL, 2011).

Tanto a PAAD quanto o megaesôfago adquirido têm caráter hereditário em cães da raça pastor alemão e variantes da raça (MENZEL \& DISTL, 2011). O diagnóstico das duas alterações baseia-se na anamnese compatível e no achado do exame radiográfico contrastado, apresentando dilatação do corpo esofágico cranial à base do coração (FARROW, 2003; ETTINGER \& FELDMAN, 2004), e o tratamento deve ser cirúrgico e instituído o mais precocemente possível (KYLES, 2012; RADLINSKY, 2013).

Assim, o presente estudo objetivou relatar a ocorrência de megaesôfago secundário associado à PAAD em dois cães da raça pastor alemão e pastor canadense. 


\section{RELATO DE CASOS}

Foram atendidos dois cães filhotes das raças pastor canadense e pastor alemão no Hospital Universitário de Medicina Veterinária da Universidade Federal Fluminense (HUVET-UFF), com queixa principal de regurgitação pós-prandial.

Primeiro caso: Cão, da raça Pastor canadense, dois meses de idade e 3,3kg de peso corporal, com histórico de emagrecimento progressivo, regurgitação pósprandial e sem alteração no apetite por sete dias. O exame clínico revelou baixo escore corporal (2/5).

Segundo caso: Cadela, da raça pastor alemão, dois meses de idade e $3 \mathrm{~kg}$ de peso corporal, com histórico de apetite voraz e regurgitação pós-prandial iniciada logo após o desmame. O exame clínico revelou alteração na ausculta pulmonar (ruídos descontínuos ásperos).

Em ambos os casos, procedeu-se a exames complementares (hematológico e exames de imagem). $O$ pastor canadense foi o que apresentou mais alterações hematológicas, com leucocitose global (de $53.700 / \mu \mathrm{L}$ ) por neutrofilia com desvio nuclear de neutrófilos à esquerda.

O ecocardiograma dos pacientes revelou normalidade na hemodinâmica cardíaca. As radiografias torácicas simples e contrastada revelaram megaesôfago cranial à base cardíaca, sugerindo anomalia de anel vascular com compressão esofágica, em ambos os casos, além de pneumonia aspirativa possivelmente secundária ao megaesôfago, mais severa no caso do pastor canadense (Figura 1).

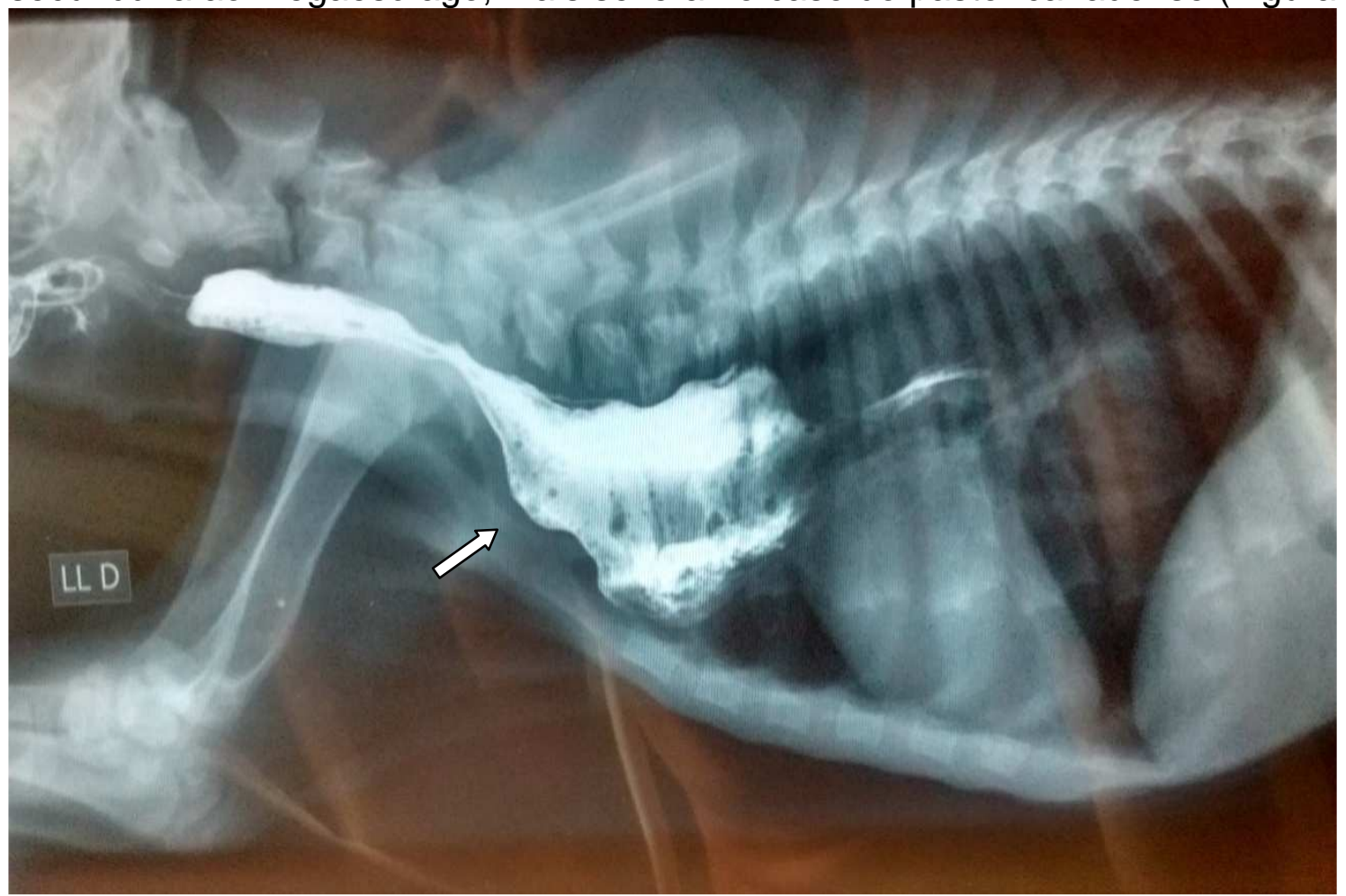

FIGURA 1. Exame radiográfico contrastado de esôfago de canino macho, pastor canadense, dois meses de idade, com vista lateral esquerda mostrando dilatação esofágica cranial à base cardíaca (seta). Fonte: Arquivo pessoal. 
Baseado na anamnese e nos resultados dos exames complementares, foi concluído o diagnóstico de megaesôfago adquirido secundário à anomalia do anel vascular. Recomendou-se o tratamento cirúrgico, mas, por motivos financeiros, os responsáveis pelos animais não permitiram o procedimento e tampouco puderam interná-los para estabilização do quadro. Além disso, não fizeram o tratamento clínico adequado, impossibilitando a cirurgia.

Nesse caso, instituiu-se, inicialmente, o tratamento da pneumonia (secundária à aspiração do conteúdo alimentar) com fluidoterapia diária e administração intravascular de antibióticos e antieméticos, além de manejo alimentar com comida pastosa na posição vertical seis vezes ao dia, até que fosse possível a intervenção cirúrgica.

Decorridos dez dias de tratamento clínico após o diagnóstico, o pastor canadense apresentou piora do quadro geral, com caquexia e emagrecimento de $1 \mathrm{~kg}$, escurecimento da cauda até a região sacrococcígea e coloração cianótica das mucosas e do abdômen (Figura 2).

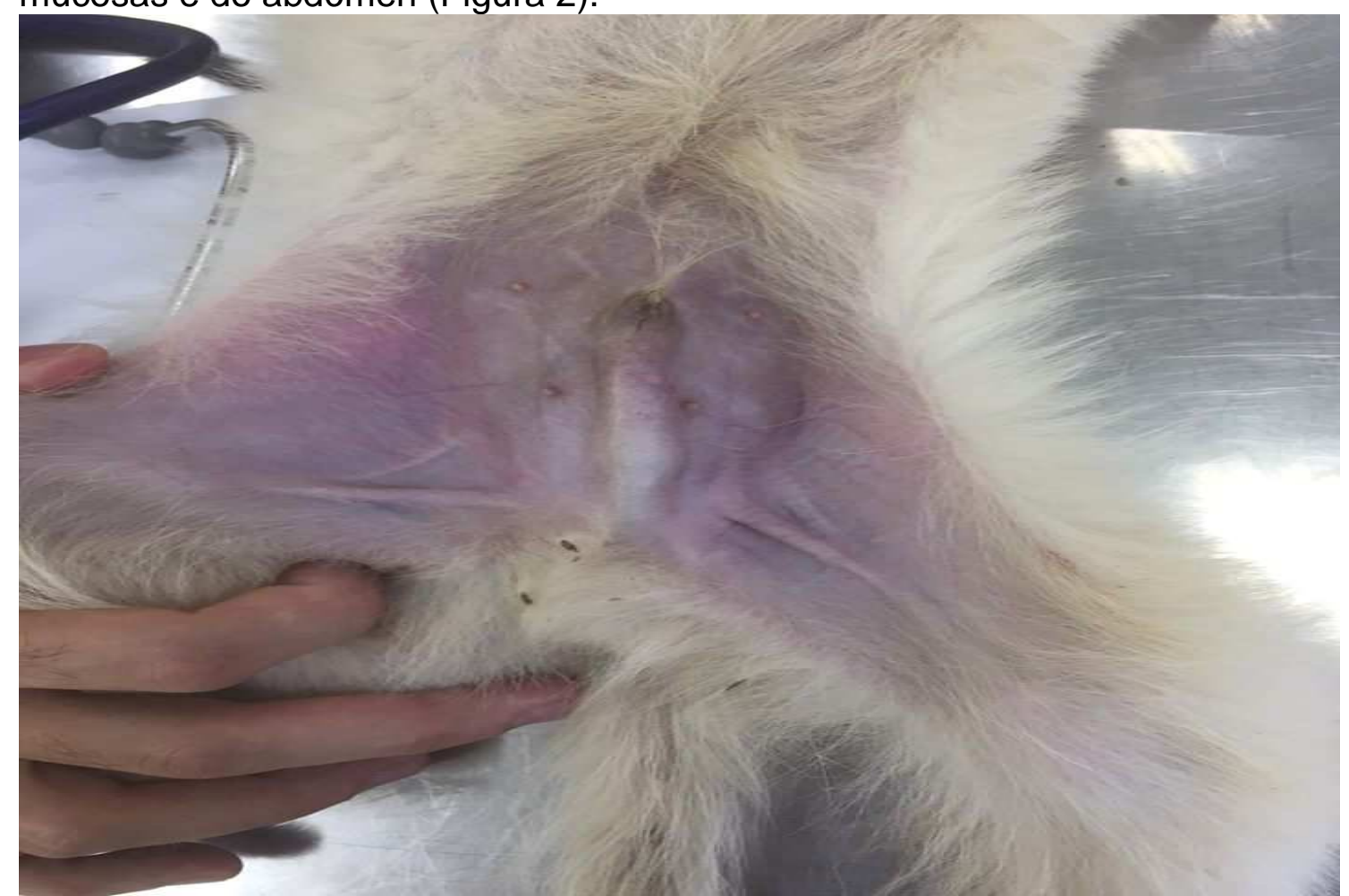

FIGURA 2. Paciente Pastor canadense com cianose em região do abdômen. Fonte: Arquivo pessoal.

O quadro continuou a se agravar, com aumento da leucocitose global (para $56.500 / \mu \mathrm{L}$ ) por neutrofilia com desvio nuclear de neutrófilos à esquerda e escurecimento também das extremidades de todos os membros do paciente, sugerindo necrose e sepse.

A paciente da raça pastor alemão também apresentou piora do quadro após 30 dias de tratamento clínico, com emagrecimento, apetite depravado, inclusive com coprofagia, e aumento das regurgitações, aproximadamente 20 por dia, pois 0 responsável não realizou o manejo alimentar adequadamente. 
Devido ao agravamento dos quadros clínicos de ambos e à impossibilidade de realizar o procedimento cirúrgico, causado pelo manejo inadequado por parte dos responsáveis, os animais foram submetidos à eutanásia. A necropsia do pastor canadense revelou o quarto arco aórtico persistente com ligamento arterioso à esquerda, causando obstrução extraluminal e dilatação esofágica cranial à base do coração (Figura 3).

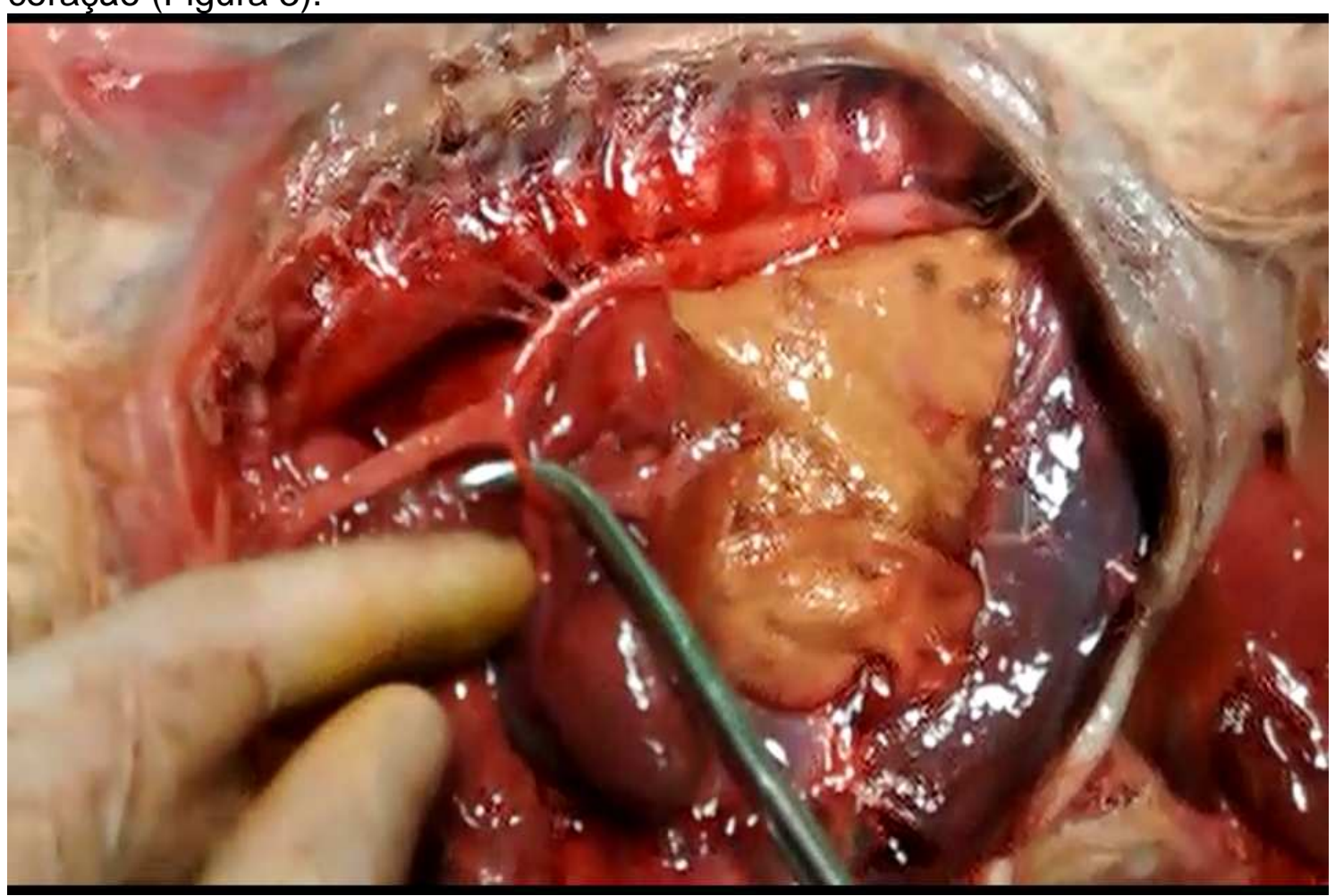

FIGURA 3. Necropsia do paciente da raça pastor canadense com persistência de arco aórtico direito com ligamento arterioso à esquerda. Fonte: Arquivo pessoal.

A necropsia da pastor alemão também revelou o megaesôfago adquirido secundário ao quarto arco aórtico direito persistente (Figura 4), exatamente como o observado na imagem da radiografia contrastada (Figura 5). 


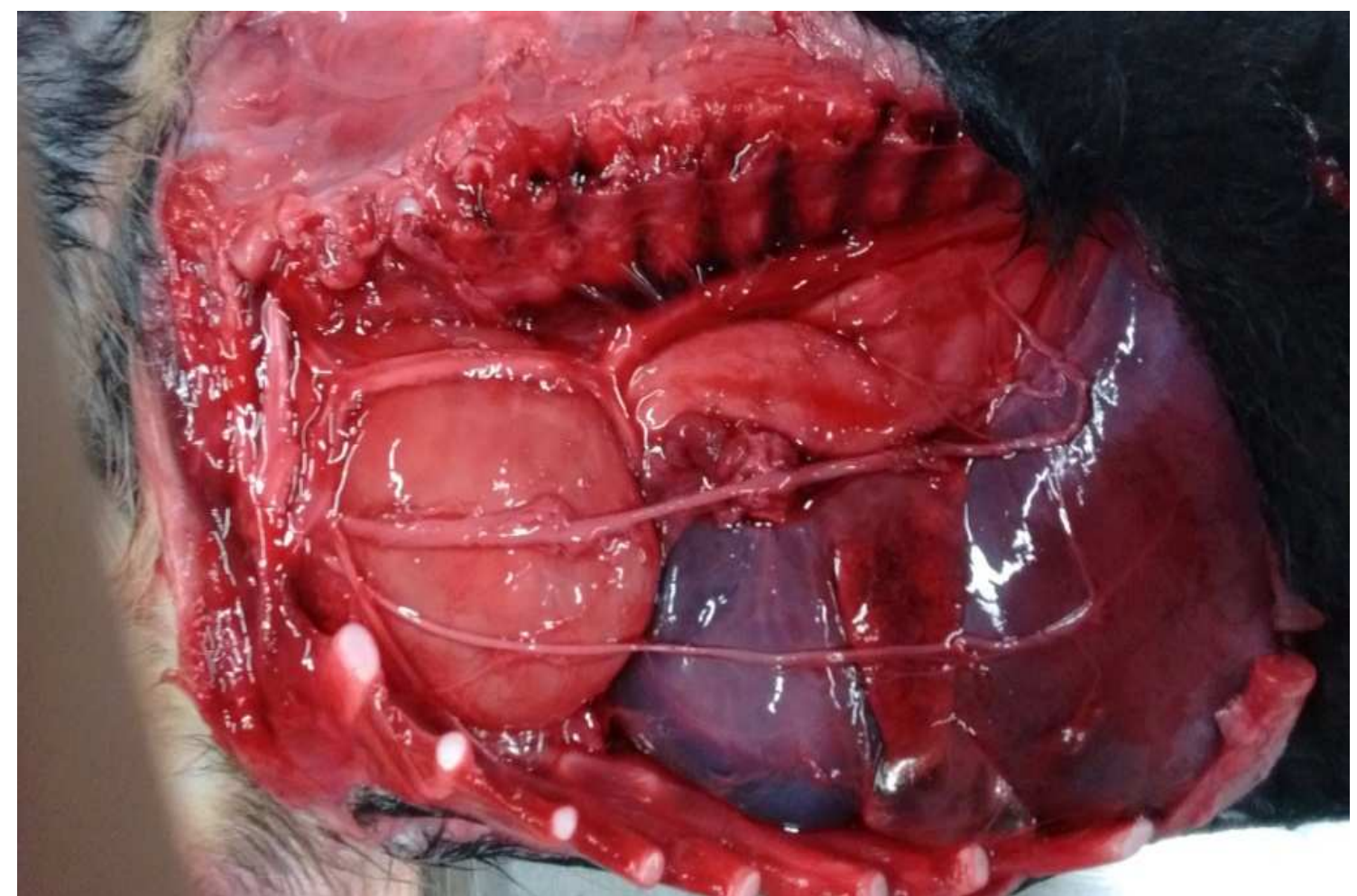

FIGURA 4. Imagem da necropsia da cadela da raça pastor alemão, vista lateral esquerda mostrando o esôfago dilatado preenchido com água via cavidade oral Fonte: Arquivo pessoal.

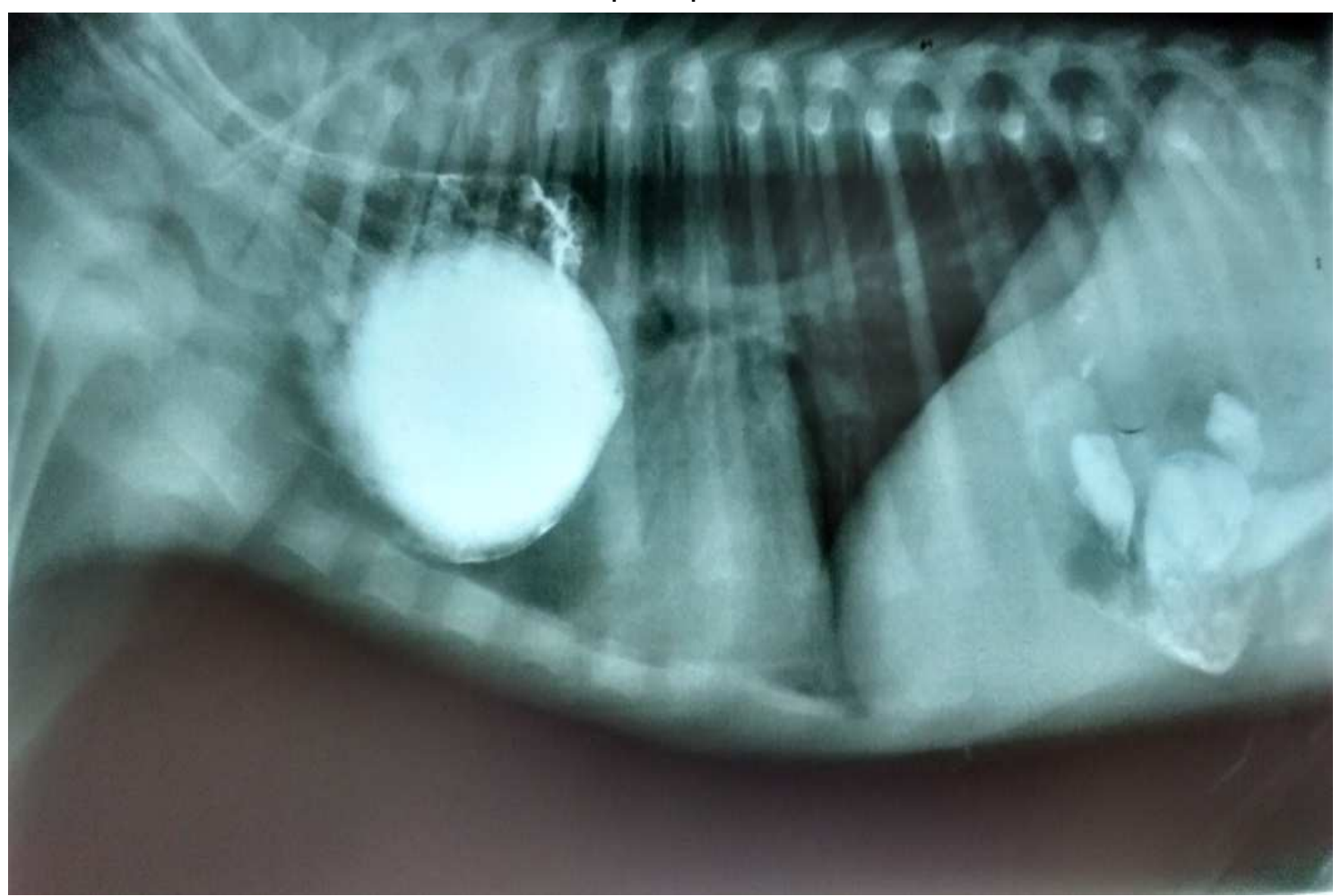

FIGURA 5. Exame radiográfico da paciente pastor alemão, uma semana antes da eutanásia. Vista lateral esquerda mostrando dilatação esofágica cranial à base cardíaca, que corrobora com a foto da necropsia. Fonte: Arquivo pessoal. 


\section{DISCUSSÃO}

Durante o desenvolvimento embrionário dos arcos aórticos, podem ocorrer anomalias na conformação dos grandes vasos cardíacos. Essas malformações ao redor do esôfago ou traqueia causam diferentes graus de constrição dessas estruturas envolvidas e são chamadas de anomalias dos anéis vasculares (MENZEL \& DISTL, 2011).

A persistência do quarto arco aórtico direito com ligamento arterioso à esquerda é a anomalia de anel vascular de maior ocorrência em cães, diagnosticada em 95\% dos casos (KOÇ et al., 2004; BOTTORFF, 2012; KREBS, 2014).

Não há evidência de predisposição sexual, afetando igualmente machos e fêmeas, como nos dois casos relatados, mas é mais comum em cães de grande porte (acima de $15 \mathrm{~kg}$ ) (KYLES, 2012) e de raças puras, sendo as mais predispostas o Boston terrier, o setter irlandês, o dogue alemão, o pastor alemão e suas variantes (QUESSADA et al., 2010; MENZEL \& DISTL, 2011). Os dados deste trabalho salientam a estatística, uma vez que os pacientes eram das raças pastor alemão e pastor canadense.

Nos dois casos, a anomalia encontrada na necropsia foi de persistência de quarto arco aórtico direito com ligamento arterioso à esquerda. Essas malformações fazem a compressão extra luminal esofágica, provocando megaesôfago secundário, geralmente com localização cranial à base cardíaca (PLESMAN et al., 2011).

O megaesôfago ocorre devido à obstrução parcial esofágica pelo anel vascular. $\mathrm{O}$ esôfago dilata gradativamente, à medida que o alimento se acumula por não conseguir passagem pelo local de compressão. Esse alimento é regurgitado e pode provocar pneumonia (SHERDING, 2013).

A pneumonia por aspiração da dieta regurgitada é relatada na maioria dos casos e deve ser tratada antes da intervenção cirúrgica (TAMS, 2003). Nos dois casos descritos neste trabalho, os pacientes apresentaram pneumonia por aspiração e foram tratados com antibióticos, mas não tiveram boa resposta devido ao manejo alimentar realizado inadequadamente pelos responsáveis, levando à progressão da pneumonia.

As regurgitações nesses casos ocorrem logo após o desmame, quando o animal passa a consumir dietas sólidas (QUESSADA et al., 2010; MENZEL et al., 2011; KREBS et al., 2014). Devido a esse distúrbio digestivo, observa-se má condição corporal dos animais, embora estes, muitas vezes, apresentem apetite voraz (BARBUR et al., 2014), o que justifica o apetite depravado, inclusive com coprofagia, da paciente pastora alemã. A regurgitação pode ocorrer logo depois ou até duas horas depois da ingestão do alimento (BARBUR et al., 2014).

Em geral, o paciente se apresenta clinicamente normal, apesar de magro, mas vai se tornando progressivamente debilitado. $O$ animal também pode apresentar tosse, dispnéia, febre e cianose (NELSON \& COUTO, 2015), como o paciente pastor canadense que, dez dias após o diagnóstico, apresentava-se acentuadamente cianótico, além de extremamente debilitado.

A PAAD não causa alteração hemodinâmica alguma no paciente (BONAGURA \& DARKE, 1997), a não ser que hajam outras anomalias cardíacas associadas, corroborando os dados deste trabalho, em que os animais não apresentaram alteração na dinâmica cardiovascular. 
Para o diagnóstico, o histórico é essencial. A sintomatologia clínica observada nos casos descritos é semelhante à citada por diversos autores (FARROW, 2003; ETTINGER \& FELDMAN, 2004; KYLES, 2012; SHERDING, 2013).

O exame radiográfico contrastado é o exame de eleição (FARROW, 2003) e foi realizado com sucesso neste trabalho. Porém, não se pode distinguir qual anomalia de anel vascular está causando a constrição (KOÇ et al., 2004), pois sua visualização e identificação só são possíveis durante o procedimento cirúrgico ou por exames complementares mais sofisticados, como a tomografia (KYLES, 2012), que não puderam ser realizados em ambos os casos deste trabalho devido à recusa dos responsáveis pelos animais.

O tratamento clínico consiste em pequenas refeições semissólidas ou líquidas oferecidas com o animal em posição vertical (NELSON \& COUTO, 2015), em um ângulo de 45 a $90^{\circ}$ em relação ao solo, e mantendo-o nessa posição por 15 minutos após a alimentação ou ingestão de água (TAMS, 2003; NELSON \& COUTO, 2015).

O tratamento cirúrgico tem como objetivo o alívio da constrição e seus consequentes sinais clínicos. É o mais indicado, visto que apenas o tratamento medicamentoso não tem bons resultados (KREBS et al., 2014).

Recomenda-se que a cirurgia seja realizada precocemente para evitar a perda da motilidade esofágica e que o megaesôfago se torne irreversível (QUESSADA et al., 2010). Nos dois casos deste trabalho, o agendamento da cirurgia foi tardio, agravando o quadro de pneumonia e caquexia dos pacientes. A literatura, no entanto, diverge quanto a isso, uma vez que a cirurgia deve ser adiada em pacientes com pneumonia por aspiração (TAMS, 2003), ao mesmo tempo que essa condição pode se agravar caso a cirurgia não seja realizada no momento devido (QUESSADA et al., 2010).

A sondagem esofágica (esofagostomia e faringostomia) é contraindicada em disfunções esofágicas primárias ou secundárias (MACPHAIL \& WILLARD, 2013), como era o caso de ambos os casos de megaesôfago adquirido secundário à anomalia do anel vascular. Há relatos de sondagem gástrica utilizada para melhorar as condições nutricionais do paciente, antes de ser submetido à cirurgia de correção da anomalia de anel vascular, com bons resultados (PLESMAN et al., 2011; MARKS, 2013; RADLINSKY, 2013), o que não se pôde tentar em virtude da rápida evolução dos quadros.

Instituído o tratamento cirúrgico, KREBS et al. (2014) relataram uma taxa de mortalidade de $8 \%$ antes da alta e uma mortalidade de $18 \%$ entre os sobreviventes. Os proprietários dos cães que sobreviveram se referem a um alto nível de satisfação. O resultado a longo prazo foi bom ou excelente em $87 \%$ dos cães, mas apenas $30 \%$ dos que sobreviveram deixaram de apresentar sinais clínicos ou de exigir novas modificações dietéticas.

Ambos os pacientes foram a óbito devido ao agravamento do quadro antes da intervenção cirúrgica, uma vez que o procedimento vinha sendo adiado em virtude da falha no manejo clínico por parte dos responsáveis, o que confirma a necessidade de realização da cirurgia o mais precocemente possível.

\section{CONCLUSÃO}

O prognóstico da PAAD é sempre reservado, devido ao risco de causar megaesôfago irreversível, pneumonia aspirativa e morte. Para reduzir a 
possibilidade de complicações, essa condição deve ser corrigida cirurgicamente o mais precocemente possível.

O tratamento clínico conservador, sem correção cirúrgica, é apenas paliativo, não sendo recomendado, conforme descrito neste trabalho, que relata o insucesso da abordagem médica sem a cirurgia, principalmente quando o manejo é inadequado por parte dos responsáveis, já que a regurgitação em geral persiste e piora à medida que o esôfago continua a dilatar. A subnutrição e a caquexia são condições que também permanecem e a pneumonia é uma ameaça constante.

\section{REFERÊNCIAS}

BARBUR, L.; MILLARD, H.T.; BAKER, S.; KLOCKE, E. Spontaneous resolution of postoperative chylothorax following surgery for persistent right aortic arch in two dogs. Journal of the American Animal Hospital Association, v. 50, n. 3, p. 209 215, 2014. Disponível em: http://dx.doi.org/10.5326/JAAHA-MS-5994. doi: 10.5326/JAAHA-MS-5994

BONAGURA, J.D.; DARKE, P.G.G. Cardiopatia congênita. In: ETTINGER, S.J., FELDMAN, E. C. (eds). Tratado de Medicina Interna Veterinária. 4ed. Brasil: Manole, 1997, p. $471-535$.

BOTTORFF, B.; SISSON, D. Hypoplastic aberrant left subclavian artery in a dog with a persistent right aortic arch. Journal of Veterinary Cardiology, v. 14, n. 2, p. 381 385, $2012 . \quad$ Disponível em: http://www.sciencedirect.com/science/article/pii/S1760273412000550 doi: 10.1016/j.jvc.2012.01.013.

DIAZ, S.A.C.; OCHOA, F.A.L.; HERRERA, H.A.P.; SARRIA, C.A.E.; HERNANDEZ, J.B. Corrección de arco aórtico derecho persistente en canino de 11 meses de edad com diagnóstico de megaesôfago. Descripción de caso clínico. Revista eletrônica de veterinária, v. 13, n. 10, p. 1 - 12, 2012. Disponível em: http://www.veterinaria.org/revistas/redvet/n101012/101215.pdf.

ETTINGER, S.J.; FELDMAN, E.C. Tratado de Medicina Interna Veterinária. 5d. Brasil: Guanabara Koogan, 2004, 2256p.

FARROW, C.S. Veterinary Diagnostic Imaging: The Dog and Cat. 1th ed. United States of America: Mosby, 2003, 772p.

GELBERG, H.B. Alimentary system and the peritoneum, omentum, mesentery and peritoneal cavity. In: ZACHARY, J.F.; MCGAVIN, M.D. (eds.) Pathologic Basis of Veterinary Disease, 5th ed, United States of America: Mosby Elsevier, 2012, p. 322 - 404. ISBN 9780-3230-7533-6

KOÇ, Y.; TURGUT, K.; SEN, I.; ALKAN, F.; BIRDANE, F.M. Persistent Aortic Arch and Its Surgical Correction in a Dog. Turkish Journal of Veterinary and Animal Science, v. 28, p. $441 \quad$ - 446, 2004. Disponível em: http://journals.tubitak.gov.tr/veterinary/issue.htm?id=523. E-ISSN: 1303-6181 
KREBS, I.A., LINDSLEY, S., SHAVER, S., MACPHAIL, C. Short- and long-term outcome of dogs following surgical correction of a persistent right aortic arch. Journal of the American Animal Hospital Association, v. 50, n. 3, p. 181- 186, 2014. Disponível em: http://www.jaaha.org/doi/abs/10.5326/JAAHA-MS6034 ?journalCode=aaha. doi: 10.5326/JAAHA-MS-6034

KYLES, A.E. Esophagus. In: TOBIAS, K.M.; JOHNSTON, S.A. (eds.) Veterinary Surgery: Small Animal. United States of America: Elsevier Saunders, 2012, p. 1461 1483.

MACPHAIL C.M.; WILLARD M.D. Nutritional management of the surgical patient. In: FOSSUM, T.W.; HEDLUND, C.S.; JOHNSON, A.L.; SCHULZ, K.S.; SEIM, H.B.; WILLARD, M.D.; BAHR, A.; CARROLL, G.L. (eds.) Small Animal Surgery, 4th ed. United States of America: Elsevier, 2013, p. 102 - 106.

MARKS, S.L. Enteral and parenteral nutrition. In: WASHABAU, R.J.; DAY, M.J. (eds.) Canine \& Feline Gastroenterology. United States of America: Elsevier Saunders, p. $429-444.2013$.

MENZEL, J.; DISTL, O. Unusual vascular ring anomaly associated with a persistent right aortic arch and an aberrant left subclavian artery in German pinschers. Veterinary Journal, v. 187, n. 3, p. $352-355$ - 2011. http://www.sciencedirect.com/science/article/pii/S1090023309004924. doi: http://dx.doi.org/10.1016/j.tvjl.2009.12.016.

NELSON, R.W.; COUTO, C.G. Anomalias do anel vascular. In: NELSON, R.W.; COUTO, C.G. Medicina Interna de Pequenos Animais. 5 ed. Rio de Janeiro: Elsevier, 2015, p. $112-114$.

PLESMAN, R.; JOHNSON, M.; RURAK, S.; AMBROSE, B.; SHMON, C. Thoracoscopic correction of a congenital persistent right aortic arch in a young cat. Canadian Veterinary Journal, v. 52, n. 10, p. 1123 - 1128, 2011. Disponível em: https://www.ncbi.nlm.nih.gov/pmc/articles/PMC3174512/pdf/cvj_10_1123.pdf.

PMCID: PMC3174512

QUESSADA, A.M.; FONTELES, Z.C.; EXPEDITA, N.A.C; RODRIGRES, M.C.; FREITAS, M.V.M.; BEZERRA, F.B.; SOUSA, J.M. Persistent Right Aortic Arch in a Dog. Acta Scientiae Veterinariae, v. 38, p. 333 - 336, 2010. Disponível em: http://www.ufrgs.br/actavet/38-3/920.pdf. ISSN 1679-9216 (Online)

RADLINSKY, M.G. Surgery of the digestive system. In: FOSSUM, T.W.;

HEDLUND, C.S.; JOHNSON, A.L.; SCHULZ, K.S.; SEIM, H.B.; WILLARD, M.D.; BAHR, A.; CARROLL, G.L. (eds.) Small Animal Surgery, 4th ed. United States of America: Mosby Elsevier, p. 386 - 583.2013. 
SHERDING, R.G. Esophagus: diagnostic evaluation. In: WASHABAU, R.J.; DAY, M.J. (eds.) Canine \& Feline Gastroenterology. United States of America: Elsevier Saunders,p. $573-580,2013$.

TAMS, T.R. Diseases of the esophagus. In: TAMS, T.R. (ed.) Handbook of Small Animal Gastroenterology, 2nd ed. United States of America: Elsevier Saunders, 2003, p. $118-158.2003$.

WASHABAU, R.J. Esophagus: obstruction. In: WASHABAU, R.J.; DAY, M.J. (eds.) Canine \& Feline Gastroenterology. United States of America: Elsevier Saunders, , p. $586-591,2013$. 